\title{
TRANSPLANTE RENAL: CONHECIMENTO DO PACIENTE ACERCA DO PERÍODO PERIOPERATÓRIO
}

\author{
Renal transplantation: awareness of patient as to the Perioperative period
}

\author{
Marcela Wanderley Rossiter ${ }^{1}$, Marília Perrelli Valença², Amanda Alves Valois ${ }^{3}$
}

\section{RESUMO}

\begin{abstract}
Objetivo: Identificar o conhecimento dos pacientes em lista de espera para realização de transplante renal atendidos no ambulatório de transplantes de um hospital escola da cidade do Recife acerca das informações e cuidados necessários no período perioperatório. Método: estudo tipo corte transversal de natureza exploratória, descritiva, quantitativa. A população foi constituída por pacientes em lista de espera do Instituto de Medicina Integral Professor Fernando Figueira - IMIP ou provenientes de outros serviços ou inscritos como casos novos para realização de transplante renal que concordaram em participar da pesquisa, maiores de 18 anos, excluindo-se aqueles pacientes que não compareceram às consultas no período de coleta de dados para o estudo. A coleta de dados foi realizada pela autora in loco por meio de entrevista semi-estruturada, com perguntas objetivas, onde foi investigado o conhecimento dos pacientes sobre transplante renal. O projeto foi aprovado pelo comitê de ética e pesquisa do Instituto de Medicina Integral Professor Fernando Figueira - IMIP, sob número do parecer: 1664. Resultados: A partir do levantamento das informações dos formulários pré-codificados foi construído um banco de dados utilizandose o programa SPSS (Statistical Package for the Social Sciences) na versão 1.5. Conclusão: Identificou-se que é deficiente o conhecimento dos pacientes acerca dos cuidados necessários no período perioperatório de transplante renal.
\end{abstract}

Descritores: Transplante de Rim; Enfermagem; Autocuidado; Orientação; Nefrologia.

\footnotetext{
Instituições:

${ }^{1}$ Departamento de Enfermagem em Clínica Cirúrgica do Instituto de Medicina Integral Professor Fernando Figueira - IMIP, Recife/PE

${ }^{2}$ Setor de Clínica Cirúrgica do Instituto de Medicina Integral Professor Fernando Figueira - IMIP, Recife/PE

${ }^{3}$ CIHDOTT do Instituto de Medicina Integral Professor Fernando Figueira IMIP, Recife/PE
}

\section{Correspondência:}

Marcela Wanderley Rossiter

Av. Isabel de Goes, 381, Areias, CEP 50870-270, Recife/PE.

Tel: (81) 3251-2828

E-mail: marcela_rossiter@hotmail.com
Aceito em: 15/01/2012

\section{INTRODUÇÃO}

Desde os primórdios, a humanidade luta para ver seus sonhos tornarem-se reais. Foi assim, sonhando com a longevidade e melhores condições de vida, que diversos pesquisadores empenharam-se na prática do transplante, palavra utilizada pela primeira vez por John Hunter em 1778, ao descrever experimentos com enxertos ovarianos e testiculares em animais. ${ }^{1}$

O transplante de órgãos e tecidos constitui-se atualmente em alternativa terapêutica segura e eficaz no tratamento de diversas doenças, determinando melhoria na qualidade e na perspectiva de vida de muitos pacientes. ${ }^{2}$

No Brasil, o primeiro transplante de órgão ocorreu em São Paulo, em 1965, com um transplante de rim. Em 1991, foi criada na Secretaria de Estado da Saúde de São Paulo uma Central de Notificação de Órgãos e Tecidos, mais conhecida como Central de Transplantes, composta inicialmente por enfermeiros. Desse modo, em muitas unidades de transplantes eram os enfermeiros que estavam incumbidos de explicar e obter das famílias o consentimento formal para a doação. ${ }^{3}$

$\mathrm{O}$ enfermeiro tem atuado em todas as equipes presentes no processo de doação: na comissão de divulgação, captação de órgãos e tecidos para transplante, nas gerências de captação e nas Comissões Intra-Hospitalares de Doação de Órgãos e Tecidos para Transplantes (CIHDOTT), tendo como finalidade desenvolver atividades no processo de identificação de pacientes e captação de órgãos, bem como elaborar rotinas e organizar atividades de orientação e conscientização para busca ativa diária de morte encefálica e parada cardiorrespiratória em âmbito hospitalar. ${ }^{4}$

O transplante renal é considerado o tratamento mais adequado para a insuficiência renal crônica; entretanto, é necessário selecionar entre os portadores de insuficiência renal crônica, aqueles que estão de acordo com os critérios, levando-se em consideração o aspecto técnico-cirúrgico, sobrevida e recidiva da doença. ${ }^{5}$ 
O número de indivíduos na fila de espera por um transplante de rim tem aumentado bastante nos últimos anos, e, apesar de o número anual de transplantes realizados também ter aumentado, apenas uma pequena porcentagem dessas pessoas chega efetivamente a ser transplantada. Segundo o Registro Brasileiro de Transplantes, até o $1^{\circ}$ semestre de 2009 havia 31.270 pacientes na lista de espera para transplante renal. ${ }^{6}$

Os candidatos a transplante têm precária qualidade de vida e vivem na perspectiva de morte iminente. A inclusão deles em uma lista de espera para realização do transplante representa a possibilidade de viver e retornar à vida normal. A possibilidade de o paciente ser submetido a um transplante pode suscitar reações contraditórias, como fascínio e inquietação. ${ }^{7}$

Entretanto, a pessoa a ser transplantada deve levar em consideração a mudança que esse procedimento proporcionará em sua vida, reinserindo-a no convívio social e reestruturando sua vida econômica, além de proporcionar a chance de voltar a executar as mesmas funções anteriores à patologia. ${ }^{8} \mathrm{O}$ paciente deve estar preparado física e psicologicamente para o transplante, devendo autorizar a entidade hospitalar a realizá-lo, através de um documento legal. ${ }^{9}$

Devido à complexidade, a modalidade terapêutica transplante renal exige que a equipe de enfermagem preste assistência específica, com qualidade e domínio técnico-científico, para embasar sua atuação. Com esse objetivo, faz-se necessário que o enfermeiro sistematize suas ações e planeje os cuidados prestados aos pacientes submetidos a transplante renal, reavaliando-os periodicamente, implementando a assistência de enfermagem e intervindo com segurança nos períodos pré, intra e pós-operatório. ${ }^{5}$

A comunicação é essencial para o relacionamento entre enfermeiro e paciente, ${ }^{10}$ bem como para a qualidade do cuidado da enfermagem. ${ }^{11} \mathrm{O}$ enfermeiro é o profissional que mais tempo permanece ao lado do paciente, sendo capaz de compreender seus diversos problemas, assim como os de seus familiares. Cabe a ele a importante função de ensinar e preparar o paciente e a família para o procedimento anestésico/cirúrgico ao qual será submetido. ${ }^{11-13}$

Para essa interação eficaz, os enfermeiros devem considerar as condições físicas, uso de medicamentos, nível cultural e aspectos psicossociais dos pacientes, ${ }^{12}$ bem como prepará-los para a cirurgia, explicando o procedimento e proporcionando suporte psicológico..$^{10,14}$

Através do processo de ensino-aprendizagem, o paciente pode adquirir conhecimentos, habilidades e ser encorajado a participar do seu tratamento, tomando decisões e assumindo responsabilidade, ${ }^{15}$ como também, alterar seu comportamento com relação à saúde. Quando ele recebe as orientações pertinentes ao seu tratamento antes da internação, sua participação ativa nos cuidados diários torna-se mais fácil, pois realiza suas atividades de autocuidado conhecendo sua importância e contando com o auxílio da enfermagem. ${ }^{10}$

Alguns trabalhos da literatura mostram os benefícios do ensino pré-operatório do paciente, tais como melhora da satisfação, menor incidência de complicações e retorno mais rápido às atividades diárias. ${ }^{16,17}$ Assim, o enfermeiro deve oferecer informações numa linguagem clara e objetiva, compatível com o nível de escolaridade e compreensão do paciente. A informação sobre a fase perioperatória pode ser realizada individualmente ou em grupos, de forma oral e com recurso audiovisual. ${ }^{12}$ Esse ensino pode influenciar nos fatores de risco e estilo de vida antes e depois da cirurgia, além de contribuir efetivamente na recuperação pós-operatória. ${ }^{16}$

Desse modo, no preparo para a cirurgia, é de extrema importância o fornecimento de orientações quanto aos procedimentos invasivos de maneira clara e objetiva e de acordo com o nível cultural do paciente, inclusive, com relação à anestesia, ventilação artificial, utilização de tubos, sondas e cateteres, monitorização cardíaca, aos exercícios respiratórios, às possibilidades de dor e administração de medicamentos. ${ }^{10,13}$

Pacientes em lista de espera sofrem a expectativa de um transplante que desencadeia sentimentos de receio, medo, dúvidas, preocupação, ansiedade e estão associados à dor, alteração da imagem corporal, dependência (dos imunossupressores) e até morte devido à grande complexidade da cirurgia. ${ }^{18}$

Eles devem compreender os motivos da necessidade de colaborar continuamente com o regime terapêutico, com atenção especial aos métodos de administração, justificativa e efeitos colaterais dos agentes imunossupressores prescritos. ${ }^{14}$ Assim, o ensino ao paciente é uma ferramenta utilizada para garantir sua independência e a detecção precoce de complicações. ${ }^{13}$

Partindo desse pressuposto, este estudo teve como objetivo identificar o conhecimento dos pacientes em lista de espera para realização de transplante renal acerca das informações e cuidados necessários no período perioperatório.

\section{MÉTODOS}

Trata-se de um estudo tipo corte transversal de natureza exploratória, descritiva, quantitativa. A pesquisa quantitativa envolve a coleta sistemática de informação numérica mediante condições de controle, além da análise dessa informação, utilizando procedimentos estatísticos. ${ }^{19}$ A pesquisa descritiva objetiva descobrir e observar fenômenos a partir de variáveis, a fim de descrevê-los, classificá-los e interpretá-los. ${ }^{20}$ Um trabalho é de natureza exploratória quando envolve algum fenômeno de interesse, levantamento bibliográfico, entrevistas com pessoas que tiveram (ou têm) experiências práticas com o problema pesquisado e análise de exemplos que estimulem a compreensão. No entanto, mais do que simplesmente observar e descrever o fenômeno, a pesquisa exploratória investiga sua natureza complexa e outros fatores com os quais ele está relacionado. Possui ainda a finalidade básica de desenvolver, esclarecer e modificar conceitos e idéias para a formulação de abordagens posteriores. ${ }^{21}$

A amostra foi composta por 98 pacientes que se encontravam no processo de admissão na lista de espera de transplante renal de um hospital escola da cidade do Recife e que se dispuseram a participar do estudo.

Foram utilizados como instrumentos para coleta de dados, questionários com perguntas abertas e fechadas às variáveis do estudo. Todos os questionários foram preenchidos com a presença da pesquisadora no momento, que orientava os pacientes sobre quaisquer dúvidas relacionadas às perguntas. No caso de pacientes analfabetos, a pesquisadora leu e preencheu o questionário para o paciente de acordo com suas respostas, e, em seguida, o termo de consentimento era assinado pelo responsável pelo paciente ou era marcada sua digital no termo. A coleta de dados foi realizada no período de março a julho de 2010. Os questionários são compostos por duas partes: dados de identificação e conhecimentos específicos sobre o tema.

\section{Aspectos Éticos}

O estudo foi autorizado pelo Comitê de Ética em Pesquisa do Instituto de Medicina Integral Professor Fernando Figueira- IMIP, visando a autorização da coleta de dados, publicação e apresentação dos resultados da pesquisa em eventos científicos.

$\mathrm{O}$ projeto foi aprovado pelo comitê de ética em pesquisa sob o número do parecer: 1664 .

Todos os participantes assinaram o Termo de Consentimento 
Livre e Esclarecido, conforme determina a Resolução no 196/96 que trata da pesquisa com seres humanos, para participação dos sujeitos no estudo, no qual, foi assegurada a confidencialidade das informações.

\section{Processamento e análise de dados}

Os dados obtidos com o grupo total de pacientes foram submetidos à análise, sendo apresentados através de gráficos e tabelas, $\mathrm{Na}$ análise dos dados foram obtidas distribuições absolutas e percentuais e as medidas estatísticas média, mediana e desvio padrão e foram utilizados os testes estatísticos: Qui-quadrado de Pearson ou Exato de Fisher quando as condições para utilização do teste Qui-quadrado de Pearson não foram verificadas.

A digitação dos dados foi realizada em planilha EXCEL e os cálculos estatísticos foram realizados através do programa SPSS (Statistical Package for the Social Sciences) na versão 15. A margem de erro utilizada na decisão dos testes estatísticos foi $5,0 \%$. (Tabela 1)

\section{RESULTADOS}

Tabela 1 - Distribuição dos pacientes pesquisados segundo as variáveis: faixa etária em anos, sexo, procedência, religião, estado civil e grau de instrução. IMIP - Recife, 2010:

\begin{tabular}{|c|c|c|}
\hline Variável & $\mathbf{n}$ & $\%$ \\
\hline $\begin{array}{l}\text { Faixa etária (em anos) } \\
20 \text { a } 29 \\
30 \text { a } 39 \\
40 \text { a } 49 \\
50 \text { ou mais }\end{array}$ & $\begin{array}{l}19 \\
13 \\
32 \\
34\end{array}$ & $\begin{array}{l}19,4 \\
13,3 \\
32,7 \\
34,7\end{array}$ \\
\hline TOTAL & 98 & 100 \\
\hline $\begin{array}{l}\text { Sexo } \\
\text { Masculino } \\
\text { Feminino }\end{array}$ & $\begin{array}{l}50 \\
48\end{array}$ & $\begin{array}{l}51,0 \\
49,0\end{array}$ \\
\hline TOTAL & 98 & 100 \\
\hline $\begin{array}{l}\text { Procedência } \\
\text { Recife } \\
\text { RMR } \\
\text { Interior do estado de PE } \\
\text { Outros }\end{array}$ & $\begin{array}{c}45 \\
18 \\
32 \\
3\end{array}$ & $\begin{array}{c}45,9 \\
18,4 \\
32,7 \\
3,1\end{array}$ \\
\hline TOTAL & 98 & 100 \\
\hline $\begin{array}{l}\text { Religião } \\
\text { Católica } \\
\text { Protestante } \\
\text { Espírita } \\
\text { Outras }\end{array}$ & $\begin{array}{c}63 \\
20 \\
3 \\
12\end{array}$ & $\begin{array}{c}64,3 \\
20,4 \\
3,1 \\
12,2\end{array}$ \\
\hline TOTAL & 98 & 100 \\
\hline $\begin{array}{l}\text { Estado civil } \\
\text { Solteiro } \\
\text { Casado }\end{array}$ & $\begin{array}{l}48 \\
48\end{array}$ & $\begin{array}{l}50,0 \\
50,0\end{array}$ \\
\hline TOTAL & 96 & 100 \\
\hline $\begin{array}{l}\text { Grau de instrução } \\
\text { Analfabeta } \\
\text { Fundamental incompleto } \\
\text { Ensino médio incompleto } \\
\text { Ensino médio completo } \\
\text { Superior }\end{array}$ & $\begin{array}{l}14 \\
11 \\
22 \\
29 \\
22\end{array}$ & $\begin{array}{l}14,3 \\
11,2 \\
22,4 \\
29,6 \\
22,4\end{array}$ \\
\hline TOTAL & 96 & 100 \\
\hline
\end{tabular}

A idade dos pesquisados variou de 20 a 69 anos, teve média de 45 anos, mediana de 46,00 anos e desvio padrão de +-12,94 anos.

$\mathrm{Na}$ pesquisa, são apresentadas características sócio-demográficas da amostra de pesquisados, onde destacou-se que: um pouco mais da metade $(51,0 \%)$ era do sexo masculino, correlacionando que a qualidade de vida, geralmente menor no sexo masculino determina o estado de saúde do paciente e, consequentemente, sua funcionalidade renal. Em relação à procedência, os dois maiores percentuais eram de Recife $(45,9 \%)$ ou do interior do estado (32,7\%). A maioria $(64,3 \%)$ era católica, seguido de $20,4 \%$ protestantes (evangélicos). Além disso, exatamente a metade era composta de solteiros e a outra metade, de casados. Os percentuais do grau de instrução variaram de $11,2 \%$ a $29,6 \%$, sendo o grau fundamental incompleto a maior frequência.

Outras variáveis estudadas foram: número de filhos, tipo de moradia, número de cômodos na casa, regime de ocupação da casa onde mora, número de pessoas que residem na casa, tipo de piso, tratamento da água para beber e destino dos dejetos. O maior percentual $(46,4 \%)$ dos participantes da pesquisa tinha de um a dois filhos, seguido de $29,9 \%$ que tinham três ou mais filhos. Além disso, o maior percentual $(44,3 \%)$ tinha de quatro a seis cômodos na residência, seguido de 30,9\% com sete a dez cômodos; a maioria $(77,6 \%$ ) tinha casa própria já quitada e $14,3 \%$ tinham casa alugada; em mais da metade $(56,1 \%)$ das residências, moravam de duas a três pessoas e, em $27,6 \%$, moravam quatro ou mais pessoas; na maioria $(62,2 \%)$ das casas, o piso era de cerâmica/lajota e em $35,7 \%$ o piso era de cimento; em aproximadamente $3 / 4(76,5 \%)$ da amostra, a água de beber era mineral e o restante bebia água filtrada $(13,3 \%)$ ou sem tratamento $(10,2 \%)$. O destino dos dejetos era a rede geral, sendo que, para $36,7 \%$, o destino era fossa com tampa.

Foi observado que a maioria $(97,6 \%)$ fazia hemodiálise; o maior percentual $(46,9 \%)$ fazia tratamento dialítico de um a cinco anos e $29,2 \%$, há menos de um ano. Estudos apontaram que o método de diálise mais utilizado ainda é a Hemodiálise. ${ }^{27}$

Outros aspectos nos mostraram que a maioria (67,3\%) afirmou saber o que é transplante e entre estes, a resposta mais frequente $(90,9 \%)$ foi: "É uma cirurgia de transferência de um órgão de indivíduo sadio para um indivíduo no qual o órgão não funciona mais". A maioria $(65,3 \%)$ afirmou saber quais as principais funções do rim e, entre estes, a resposta mais frequente foi: "Tirar as impurezas do sangue e também controlar a pressão arterial” (92,2\%).

Questionados se sabiam qual a principal causa de sua doença renal, a maioria $(85,7 \%)$ respondeu afirmativamente; as duas doenças mais citadas foram: hipertensão arterial sistêmica (HAS) (41,7\%) e diabetes mellitus (DM) $(13,1 \%)$.

Além disso, apenas 32,0\% afirmaram ter conhecimento sobre os procedimentos a serem realizados durante o processo de transplante e, entre estes, a resposta mais frequente foi: “O paciente irá realizar uma série de exames complementares, se necessário; também é importante ter uma dieta adequada, estar hidratado e em boas condições de saúde" (93,5\%).

Aproximadamente 3/4 (75,5\%) afirmaram saber o que é rejeição e, no referido grupo, a resposta mais freqüente foi: "É o jeito pelo qual o sistema imunológico reconhece o órgão transplantado como estranho e ativa o sistema de defesa para destruí-lo" (83,8\%), seguido da resposta "É uma reação esperada após o transplante" $(43,2 \%)$. Um pouco menos da metade $(44,9 \%)$ afirmou já ter sido orientado em relação ao estilo de vida a ser adotado no período pós-transplante e, entre esses, a orientação mais frequente foi: "O transplante de rim oferece uma chance de melhor qualidade de vida e uma independência da diálise. Assim, aquelas pessoas que fizeram transplante renal com sucesso, podem ter um dieta mais próxima do normal e ingerir líquidos de forma mais liberal. $\mathrm{O}$ mesmo pode-se dizer de sua atividade física" (97,7\%).

Em relação à questão "Você sabe quais os cuidados necessários no pós-operatório?", aproximadamente $1 / 3$ respondeu afirmativamente $(33,7 \%)$ e, destes, a maioria $(90,9 \%)$ respondeu "É importante evitar 
contado com pessoas doentes, fazer uso correto dos medicamentos e conhecer os sinais e sintomas de rejeição". O percentual que afirmou ter sido informado sobre os riscos da cirurgia de transplante foi $43,9 \%$.

Além disso, o percentual dos pacientes que afirmou ter sido orientado em relação ao estilo de vida a ser adotado no período pós-transplante reduziu com a faixa etária $(65,6 \%$ entre os que tinham até 39 anos e $30,3 \%$ entre os que tinham 50 anos ou mais), diferenças essas que revelam associação significativa entre as duas variáveis em estudo $(\mathrm{p}>0,05)$. (Tabela 2)

Tabela 2 - Avaliação da questão "Você foi orientado em relação ao estilo de vida a ser adotado no período pós-transplante" segundo a faixa etária (em anos). IMIP - Recife, 2010

\begin{tabular}{|c|c|c|c|c|c|c|c|c|c|}
\hline \multirow[t]{2}{*}{ P2 -13 } & \multicolumn{2}{|c|}{$\begin{array}{c}\text { Até } 39 \\
\text { anos }\end{array}$} & \multicolumn{2}{|c|}{$\begin{array}{c}40 \text { a } 49 \\
\text { anos }\end{array}$} & \multicolumn{2}{|c|}{$\begin{array}{l}50 \text { anos } \\
\text { ou mais }\end{array}$} & \multicolumn{2}{|c|}{$\begin{array}{c}\text { Grupo } \\
\text { total }\end{array}$} & \multirow[t]{2}{*}{ Valor de $p$} \\
\hline & $\mathbf{N}$ & $\%$ & $\mathbf{N}$ & $\%$ & $\mathbf{N}$ & $\%$ & $\mathbf{N}$ & $\%$ & \\
\hline Sim & 21 & 65,6 & 13 & 40,6 & 10 & 30,3 & 44 & 45,4 & $\mathrm{p}(1)=0,013^{*}$ \\
\hline Não & 11 & 34,4 & 19 & 59,4 & 23 & 69,7 & 53 & 54,6 & \\
\hline Total & 32 & 100,0 & 32 & 100,0 & 33 & 100,0 & 97 & 100,0 & \\
\hline
\end{tabular}

(*): Diferença significativa ao nivel de 5,0\%.

(2): Através do teste Qui-quadrado de Pearson.

O percentual de pacientes que afirmou saber o que é rejeição foi mais elevado entre os que possuiam ensino superior $(90,9 \%)$ ou ensino médio $(88,2 \%)$ e foi menos elevado entre os analfabetos $(42,9 \%)$, comprovando associação significativa entre grau de instrução e os resultados da questão $(\mathrm{p}<0,05)$.

Verificou-se, também, que a maior diferença percentual entre as categorias do tempo de tratamento dialítico em relação aos resultados da questão "Você sabe quais as principais funções do rim?" que foi de $60,9 \%$ (entre os que tinham seis anos ou mais) e $71,4 \%$ entre os que tinham menos de um ano não comprova associação significativa entre as duas variáveis em análise $(\mathrm{p}>0,05)$.

\section{DISCUSSÃO}

Observou-se que o tabagismo, etilismo e estresse, características predominantemente masculinas, são fatores estressantes e de injúria renal, o que ficou confirmado com os resultados da pesquisa. ${ }^{22} \mathrm{Em}$ relação à religião, estudos apontam que a oração e crença espiritual podem estimular o sistema imunológico e a capacidade autocuradora dos indivíduos. Além disso, as religiões moldam as percepções que as pessoas têm sobre si mesmas e também de suas respostas à dor. ${ }^{23}$

Sobre as problemáticas condição de moradia e nível de educação, foi visto que que as áreas do Estado que receberam mais investimentos do Poder Público, com instalações de boa infra-estrutura e saneamento básico são apropriadas, em sua maioria, por pessoas mais alfabetizadas e com grau de instrução e renda maiores. As condições de moradia nesses locais são obviamente mais favoráveis. A população com grau de instrução e renda menores apropria-se de locais que não dispõem de saneamento adequado, tanto na capital quanto no interior, expondo-se a riscos decorrentes das precárias condições de moradia, principalmente com manuseio inadequado de água. A falta de canalização interna leva as pessoas a armazenar água no domicílio de forma incorreta, facilitando sua contaminação ou propiciando ambientes favoráveis à proliferação de mosquitos, particularmente do Aedes aegypti. A falta de instalações sanitárias piora o quadro, pois sem um meio adequado para despejo dos dejetos há maior facilidade para disseminação de doenças infectoparasitárias. $^{24}$
Sabe-se, também, que a educação é importante na formação de novas crenças, processo que acontece nas relações estabelecidas e que podem mudar as emoções, propiciando nova visão do tratamento. ${ }^{25}$

Sobre as relações e conflitos inter-pessoais, observou-se que a família de paciente com doença terminal convive com sentimentos variados, que vão desde atitudes de hostilidade até atos de superproteção, amor, preocupação e culpa. Portanto, a família tem grande influência no tratamento de doença crônica, sendo importante sua orientação e instrução sobre todo o processo do cuidar, já que é tida como facilitadora para o paciente obter apoio emocional. ${ }^{26}$

Muitos estudos reafirmam que os indicadores de condições de moradia representados pelo número médio de cômodos, banheiros, e de pessoas por domicílio são importantes, pois sabe-se que algumas doenças contagiosas como turbeculose e hanseníase, patologias de extrema gravidade para pacientes transplantados (imunossuprimidos), têm como fatores de risco ambientes inapropriados para o convívio de pessoas. ${ }^{24}$

Em relação à questão do conhecimento sobre as causas que acarretaram a insuficiência renal crônica (IRC), sabe-se que vão desde doenças primárias renais até doenças sistêmicas e do trato urinário. A nefropatia diabética, hipertensão e glomerulonefrite primária são as causas mais comuns da insuficiência renal terminal, seguidas de lesões hereditárias (doença renal policística), mieloma, infecções, medicamentos, agentes tóxicos, agentes ambientais e ocupacionais (chumbo, cádmio, mercúrio e cromo). ${ }^{28}$

Portanto, o conhecimento sobre a patologia e como participar ativamente do tratamento favorecem os sentimentos de controle da situação, sendo considerado fator fundamental para manutenção do equilíbrio emocional e da motivação do paciente para cumprir o tratamento médico. ${ }^{29}$

Assim, os pacientes renais crônicos em fila de espera para transplante renal acabam tornando-se desanimados, desesperados e, muitas vezes, por essa razão, ou por falta de orientação, abandonam o tratamento ou não dão importância aos cuidados constantes que deveriam ter. É importante e necessário estimular suas capacidades para que se adaptem ao novo estilo de vida e assumam o controle do seu tratamento. ${ }^{30}$

Dada tal importância à compreensão do processo, observa-se que os jovens, hoje em dia, têm maior habilidade e acesso a maiores informações sobre os mais variados temas, comparados aos indivíduos mais velhos.

Sendo assim, é de extrema importância a orientação a todos os pacientes sobre o processo do transplante, pois os sentimentos de controle da enfermidade fortalecem a autovalorização do paciente e trazem, consequentemente, sentimentos de segurança e confiança. $\mathrm{O}$ desconhecimento e a incerteza sobre o tratamento causam ansiedade, agressividade e conduta inapropriada frente a ele, fazendo com que o paciente deixe de lutar e perca a esperança. ${ }^{29}$

Sabe-se que, independentemente, do tempo de hemodiálise, muitos não têm conhecimento sobre informações básicas a respeito de seu tratamento e sua patologia. Isso evidencia, mais uma vez, a carência de informações disponibilizadas para esses pacientes pelos profissionais de saúde, deixando-os à mercê das condutas médicas como meros "objetos para realização de procedimentos".

\section{CONCLUSÃO}

Apesar desses resultados restringirem-se ao local onde foi realizada a pesquisa, eles são um indicativo da importância do trabalho da equipe de enfermagem que assiste o paciente em lista de espera para transplante renal, o qual apresenta expectativas e almeja a realização de tal procedimento, misturando euforia com insegurança e medo. 
Além da relevância da orientação educacional do paciente, é importante que o profissional cultive uma imagem positiva sobre seu trabalho, o que vai influenciar sua conduta e suas emoções frente a situações de dor, sofrimento e até morte, tornando-o uma fonte de prazer e realização pessoal.

A educação adequada do paciente faz com que a responsabilidade sobre o tratamento seja compartilhada com ele, devendo sempre receber informações sobre as fases e mudanças do seu tratamento, para que se torne ativo no decorrer deste. Com isso, as percepções dos pacientes deixam de ser idealistas e tornam-se realistas, ajudando no desenrolar de todo o processo do transplante.

Observou-se que muitos pacientes, quando questionados sobre a principal causa de sua doença renal, responderam, em sua grande maioria, causas relacionadas à $\mathrm{HAS}, 41,7 \%$, e a DM, $13,1 \%$, mostrando com isso o alto grau de comorbidade daquelas patologias, principalmente acometimento renal, e da importância de atividades preventivas e educativas, assim como do seu controle pela população.

Outro aspecto observado foi que apenas 32,0\% afirmaram ter conhecimento sobre os procedimentos a serem realizados durante o processo de transplante, reforçando mais uma vez a importância da função educacional do enfermeiro ao transmitir conhecimento e trocar experiências, fazendo com que o paciente seja o ator principal de sua própria história e tenha controle da evolução do seu tratamento.

Com esse resultado, fica evidente a necessidade de acompanhamento dos pacientes nesse momento tão específico de suas vidas, onde estão à espera de um transplante, vislumbrando novas possibilidades, porém temerosos em relação a um futuro incerto. A dependência da diálise para sobreviver, além do enfrentamento das complicações que podem advir com a doença renal, são desafiadoras, remetendo o paciente ao confronto com a morte e a descrença, ao mesmo tempo em que busca força e fé para aguardar o surgimento de um doador.
A atuação da enfermagem é fundamental nesse processo, devendo mostrar disponibilidade ao diálogo, ouvir e estar junto de cada paciente, construindo uma forma de cuidado humanizado $\mathrm{e}$ integral.

Além disso, aproximadamente 3/4 (75,5\%) afirmaram saber o que é rejeição e, no referido grupo, $43,2 \%$ responderam que seria a reação esperada após o transplante, ficando muitos em dúvida sobre a existência de tratamento para tal episódio, fortificando ainda mais a idéia da importância da atuação educativa da enfermagem junto aos pacientes.

Outro ponto notado foi em relação à questão do conhecimento sobre os cuidados necessários após o transplante e sobre as informações dadas pela equipe médica sobre os riscos da referida cirurgia, sendo que menos da metade entrevistada, 33,7\%, respondeu que tinha conhecimento sobre os cuidados no pós-operatório e 43,9\% respondeu ter sido orientada sobre os riscos do procedimento. Para a realização de boas escolhas, é necessário que o paciente esteja ciente de sua situação, e seja informado sobre todas as possibilidades, vantagens e desvantagens de cada uma, como também que a equipe médica não imponha qual o melhor caminho a ser seguido dentro de suas possibilidades específicas, obviamente. Todos os momentos devem ser aproveitados para a exploração dessas possibilidades de escolha, criando condições de mudanças, quando e onde forem necessárias, na busca de melhor qualidade de vida, apesar da doença.

Por esses e tantos outros números e relatos, observou-se que há muito o que evoluir no tripé enfermeiro-paciente-comunicação, talvez pela ausência da consulta de Enfermagem no serviço. É papel do enfermeiro mostrar-se seguro e manter um bom relacionamento interpessoal, acolhedor e de confiança, de modo a complementar a intervenção terapêutica. Só assim existirá a possibilidade de traçar uma linha de ação conjunta com o paciente, encontrando soluções para problemas detectados, contribuindo dessa forma para a promoção, manutenção e recuperação da saúde física e mental dos pacientes.

\section{ABSTRACT}

Purpose: This study aims to identify the knowledge of patients on waiting lists for kidney transplant surgery at an outpatient transplant clinic of a school hospital in Recife as to the information about the care required along the perioperative period. Method: a cross sectional study using a exploratory, descriptive and quantitative method. Population will be constituted of patients on waiting lists at the school hospital in Recife or from other services recorded as new cases of kidney transplantation surgery who agree to participate in the survey, ages above 18 years, excluding patients who did not come out during the data collection for the survey. Data collection was performed by the author in situ through semi-structured interviews, composed by objective issues investigating the patients' knowledge about kidney transplantation. The project was approved by the ethics committee and research of Institute of Integrative Medicine Prof. Fernando Figueira - IMIP, through the statement no.: 241/09. Results: From the information collected in the survey with pre-coded forms, it was mounted a database using the SPSS (Statistical Package for the Social Sciences) software version 1.5, and it was found the frequency distribution of variables presented as tables. Conclusion: It was identified that the patients' knowledge on the care required along the perioperative period of kidney transplantation is inapropriate.

Keywords: Kidney Transplantation; Nursing; Self Care; Orientation; Nephrology. 


\section{REFERÊNCIAS:}

1. Pereira WA. Manual de transplantes de órgãos e tecidos. Rio de Janeiro: Medsi, 1996.

2. Ministério da Saúde (Brasil). 2006.

3. Oquisso T, Schmdt MJ. O exercício da Enfermagem: Uma abordagem éticolegal. São Paulo/SP: LTr; 1999:102-3.

4. Celitte I, Maia L, Silva M, Cardinal H. Papel da Enfermagem nas equipes de transplante de Cuiabá/MT. Anais do 9. Congresso Brasileiro de Enfermagem, 24 a 28 de setembro de 2006, Porto Seguro : COFEN, 2006.

5. Pereira W.A. Manual de transplantes de órgãos e tecidos. $3^{\circ}$ ed. Rio de Janeiro: Guanabara Koogan, 2004;268-96.

6. Associação Brasileira de Transplantes de Órgãos. Protocolo de cuidados de enfermagem em transplante de órgãos. Assistência de Enfermagem ao paciente submetido a transplante renal. 2008.

7. Massarollo MC, Kurgant P. Nursing perceptions in the liver transplantation program of a public hospital in Brazil. In: NURSING Revista Técnica de Enfermagem: Assistência de Enfermagem em Transplante: a importância do enfermeiro nesta modalidade terapêutica. Maio, 2003;60:16-20.

8. Gilliam JE. Roles of the liver transplante clinical nurse specialist. Clin Nurse Spec 5:73-77, 1991. In: NURSING Revista Técnica de Enfermagem: Assistência de Enfermagem em Transplante: a importância do enfermeiro nesta modalidade terapêutica, 1991;60;16-20.

9. Ferrarini CDT, Muller ML, Mello MZR. Transplante de coração Enfermagem. In: Revista Brasileira de Enfermagem.1968;21(6):529-37.

10. Zago MMF, Casagrande LDR. A comunicação do enfermeiro cirúrgico na orientação do paciente: a influência cultural. Ver Latino-am Enfermagem 1997;5(4):69-74

11. Bensing JM, Visser A, Saan H. Patient education in the Netherlands. Patient Educ Couns 2001;44(1):15-22.

12. Saarmann L, Daugherty J, Riegel B. Patient teaching to promote behavioral change. Nurs Outlook. 2000;48(6):281-7.

13. Smeltzer SC, Bare BG. Avaliação e conduta de pacientes com distúrbios hepáticos e biliares. In: Smeltzer SC, Bare BG, organizadores. Brunner/ Suddarth Tratado de enfermagem médico-cirúrgica. 7aed. Rio de Janeiro (RJ): Guanabara Koogan; 1994;831-72.

14. Cerezo MCM, Simón CS, Muñoz MP, Romanillos MTA, Agustí IT, Canales PB. Estudio del proceso educativo en el paciente con trasplante hepático. Enf Intensiva 2001;12(2):58-65.

15. Zago MMF. Considerações sobre o ensino do paciente cirúrgico. Rev Esc Enfermagem USP 1993;27(1):67-71.
16. Brumfield VC, Kee CC, Johnson JY. Preoperative patient teaching in ambulatory surgery settings. Aorn J 1996;64(6):941-6,948,951-2.

17. Johansson K, Salanterä S, Katajisto J, Leino-Kilpi H. Patient education in orthopaedic nursing. J Orthop Nurs 2002;(6):220-6.

18. Sasso K, Galvão C, Silva Jr O, França A. Transplante de fígado: resultados de aprendizagem de pacientes que aguardam a cirurgia. Rev. Latino-Am. Enfermagem. 2005;13(4).

19. Polit DF, Hungler BP. Fundamentos de pesquisa em enfermagem. $3^{\mathrm{a}}$ ed. Porto Alegre (RS): Artes Médicas, 1995.

20. Vieira V. As tipologias, variações e características da pesquisa de marketing. Rev. FAE. 5(1)61-70.[s.d.]

21. Polit DF, Beck CT, Hungler B P. Fundamentos de Pesquisa em Enfermagem Métodos, avaliação e utilização. $5^{\circ}$ ed. Porto Alegre: Artmed. Exploração da Pesquisa em Enfermagem; 2004;34-5.

22. Azevedo BAR. A qualidade de vida dos pacientes portadores de insuficiência renal crônica submetidos à hemodiálise avaliada pelo instrumento genérico Sf-36. Rev. Medicina Alternativa. 2010.

23. Domar AD, Drherer H. Equilíbrio mente/corpo na mulher: uma abordagem holística para administrar os estresse e assumir o controle de sua vida. $2^{\circ}$ ed. Rio de Janeiro: Campus, 1997.

24. Pontes CAA, Costa AM, Gonçalves FR. Análise espacial da distribuição dos serviços básicos de saneamento: Desigualdade intra-urbana no Recife - PE. XXVII Congresso Interamericano de Engenharia Sanitária e Ambiental. 2000.

25. Maturana HR. Emoções e linguagem na educação e política. Belo Horizonte:Editora UFMG, 1999.

26. Atkinson RL, Atkinson RC, Smith EE, Bem DJ, Hoeksema SN. Introdução à Psicologia de Hilgard. $13^{\circ}$ ed., Porto Alegre: Artmed, 2002.

27. Smeltzer SC, Bare BG, Hinkle JL, Cheever KH. Tratado de Enfermagem em Médico- Cirúrgica - Brunner \& Suddarth. $11^{\circ}$ ed., Rio de Janeiro: Guanabara, 2008.

28. Ribeiro RCHM, Oliveira GASA, Ribeiro DF, Bertolin DC, Cesarino CB, Lima LCEQ, Oliveira SM. Caracterização e etiologia da insuficiência renal crônica em unidade de nefrologia do interior do Estado de São Paulo. Acta Paul Enferm 2008;21(Número Especial):207-11.

29. Garcia AL. Estrés psicológico y enfrentamento: Uma actualización del tema (Em Imprenta). 2000.

30. Lima AFC, Gualda DMR. O significado da hemodiálise para o paciente renal crônico: utilização de um modelo para interpretação de dados de pesquisa qualitativa. Rev Paul Enf. 2001(maio);20(2)44-51. 